\title{
CONCEITOS DE ERGODICIDADE E AUTOCORREÇÃO APLICADOS AO FILTRO DE WIENER
}

\author{
R. D. $\operatorname{COSTA}^{1,2^{*}}$, R. A. M. VALENTIM ${ }^{2}$, A. D. DÓRIA NETO ${ }^{2}$, H. J. S. CASTRO ${ }^{2}$ e J. W. O. SILVA ${ }^{3}$ \\ ${ }^{1}$ Instituto Federal de Educação, Ciência e Tecnologia do Rio Grande do Norte - IFRN \\ ${ }^{2}$ Programa de Pós-graduação em Engenharia Elétrica e de Computação - UFRN \\ ${ }^{3}$ Programa de Pós-graduação em Engenharia Mecatrônica - UFRN \\ douglas.costa@ifrn.edu.br
}

Submetido 29/02/2016 - Aceito 14/06/2017

DOI: $10.15628 /$ holos.2017.4186

\section{RESUMO}

Atualmente, diferentes técnicas têm sido desenvolvidas para tratamento de sinais com o objetivo da remoção de ruídos existentes, principalmente em sinais de voz. Muitas aplicações têm sido desenvolvidas e aperfeiçoadas nas mais diversas áreas tecnológicas, como as de telecomunicações, automação, eletrônica entre outras. Problemas como o de interferência inerente de um sistema, seja ele na detecção, no processamento, na transmissão ou na recepção, tem sido motivo para o aprimoramento de técnicas de otimização. Com o objetivo de restaurar os sinais de qualquer natureza, no qual sofreram algum tipo de degradação ou distorção, foi investigado o uso de filtros de Wiener através de algoritmos implementados em "Matlab", através de uma abordagem conceitual de teorias de processos estocásticos baseados nos princípios de ergoticidade, autocorrelação e equações de Yule-Walker.

PALAVRAS-CHAVE: Ergodicidade, Autocorreção, Processos Estocásticos, Filtro de Wiener.

\section{ERGODICITY AND AUTOCORRECT CONCEPTS APPLIED THE WIENER FILTER}

\begin{abstract}
Currently, various techniques have been developed for the treatment of signs for the purpose of removing existing noise, especially in voice signals. Many applications have been developed and refined in several technology areas, such as telecommunications, automation, electronics, among others. Problems such as interference inherent in a system, be it in detection, processing, transmission or reception has been reason
\end{abstract}

for the improvement of optimization techniques. In order to restore the signals of any nature, in which suffered some type of degradation or distortion, the use of Wiener filters using algorithms implemented in Matlab was investigated, through a conceptual approach of theories of stochastic processes based on the principles of ergodicity, autocorrelation and YuleWalker equations.

KEYWORDS: Ergodicity, Autocorrect, Stochastic Processes, Wiener Filter 


\section{INTRODUÇÃO}

Hoje, devido ao progresso tecnológico e a ascensão dos meios de comunicação, exige-se que as mensagens sejam transmitidas cada vez mais rápida e sem perdas.

Nesse intuito, o processamento digital de sinais procura melhorar a qualidade do sinal difundido no processo de comunicação entre transmissor e o receptor, através do uso de filtros adaptativos (Sarmento, Regis \& Araújo, 2012).

Nos últimos anos tem-se constatado um grande crescimento de técnicas de controle ativo de ruído através do uso de filtros adaptativos com intuito de melhora cada vez mais a comunicação digital. Esse tipo de comunicação possui vantagens de compatibilidade física, manuseio e reprodução fácil, em relação a comunicação analógico, pois nesta era necessária a construção de circuitos eletrônicos para realizar tais operações (Silva, 2009).

Neste trabalho, será usado o filtro de Wiener, que é um tipo de filtro adaptativo, com a finalidade de recuperação de um sinal de áudio que sofreu distorções por um ruído aditivo indesejado.

O ruído corresponderá a um sinal de ruído branco com distribuição normal, com uma variância igual a 1 (um). Será calculado através da diferença entre um sinal de referência e a saída do filtro.

O artigo está organizado da seguinte forma: A Seção 2 apresenta a fundamentação teórica do trabalho, apresentando os conceitos de ruído e seus tipos, sinal e filtragem de sinais estocásticos, como também os conceitos de processos estocásticos estacionários e ergódicos e as função de correção cruzada e auto correlação e o conceito dos filtros FIR de Wiener. Na seção 3 é apresentado o modelo computacional desenvolvido neste trabalho e o seu resultado mostrado na seção 4. E por fim, as conclusões na seção 5.

\section{FUNDAMENTAÇÃO TEÓRICA}

\subsection{O ruído}

O ruído é um sinal interferente de natureza aleatória que provoca a degradação de sinal durante seu processamento. Ou ainda, pode-se considerar um ruído como sendo qualquer fonte de erro, distúrbio ou deformação da fidelidade na comunicação de uma mensagem (Oliveira, 2007).

O controle de ruído ativo foi inicialmente patenteado por "Paul Lueg" na Alemanha em 1932 (Lueg, 1932) e continua sendo desenvolvido e aperfeiçoado por vários pesquisadores, de forma bastante acelerada, até os dias de hoje.

O Ruído é quantificado através de sua potência e pode ser classificado pelo seu tipo, pela frequência em que atua, e pela sua origem. Como por exemplo:

Ruído Térmico ou de Johnson-Nyquist: Gerado devido à agitação térmica dos átomos. Um dos casos mais característicos de ruído térmico é aquele gerado pelos resistores metálicos. Quanto 
maior a temperatura, maior será esse ruído. Este ruído é quase que um ruído branco (SANT'ANNA, 2008).

Ruído Shot: Causado pelo fluxo finito de partículas (elétrons ou fótons). O elétron possui carga discreta, logo o fluxo de elétrons possui carga discreta e a circulação dos mesmos gera uma flutuação do campo elétrico e por consequência uma flutuação da corrente elétrica. Tendo em vista que o circuito eletrônico sempre haverá fluxo de elétrons durante seu funcionamento, podemos dizer que é impossível ser eliminado (Oliveira, 2007).

Ruído Rosa, Ficker ou 1/f: ocorre em quase todos os dispositivos eletrônicos, e para uma frequência " $\mathrm{f}$ " constante, sua potência cai cerca de $3 d B$ por oitava. Para frequências altas o suficiente, este ruído nunca é o dominante, devido à atenuação da sua potência. São várias as caudas do ruído rosa, podemos citar a mudança de valor das resistências dos componentes (lembrando que a resistência de um material varia com a sua temperatura). Os tubos de raios catódicos, quando emitindo corrente é outro exemplo (Filho, 2009).

Ruído branco: Por definição aquele que tem a sua potência distribuída uniformemente no espectro de frequência, ou seja, $\mathrm{Sw}(\mathrm{f})=\mathrm{Nw}$ é uma constante. O nome ruído branco advém da analogia com o espectro eletromagnético na faixa de luz. A luz branca contém todas as frequências do espectro visível (Filho, 2009).

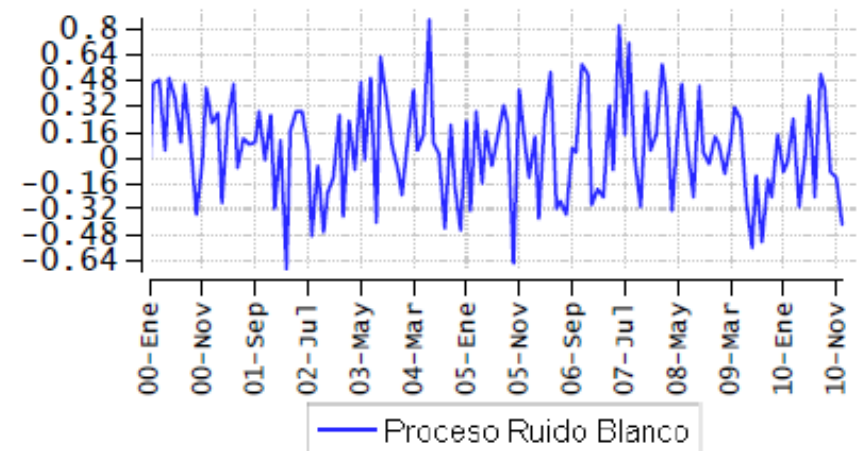

Figura1: Exemplo de um ruído branco (DAVILA; 2009)

\subsection{O sinal}

O sinal é uma função que transmite informação sobre um fenômeno físico. O qual pode ser classificado em: sinal de tempo contínuo, sinal de tempo discreto, sinal par, sinal ímpar, sinal aleatório, determinístico, sinal de energia, sinal de potência, sinal periódico e sinal não periódico (Sarmento et al., 2012).

Neste trabalho, o sinal desejado ou sinal de referência (d) possui uma frequência sinusoidal de 0,45 e 200 amostras, este sinal é desejado para tratar um sinal de ruído branco com distribuição normal, com variância igual 1 (um).

\subsection{Filtragem de sinais estocásticos}

O processo de filtragem de sinais estocásticos consiste na análise e/ou modificação de sinais utilizando teoria fundamental, aplicações e algoritmos, de forma a extrair informações dos mesmos e/ou torná-los mais apropriados para alguma aplicação específica. 
Este processo utiliza matemática, estatística, computação, heurística e representações linguísticas, formalismos e técnicas de representação, modelagem, análise, síntese, descoberta, recuperação, detecção, aquisição, extração, aprendizagem, segurança e forense.

\subsection{Processos estocásticos estacionário}

Dizemos que um processo é estacionário se todas as características do comportamento do processo não são alteradas no tempo, ou seja, o processo se desenvolve no tempo em torno da média, de modo que a escolha de uma origem dos tempos não é importante. (Portal Action, 2015).

Num processo estacionário as médias conjuntas são independentes do tempo de observação, como se pode observar na fórmula abaixo:

$E\left\{x^{m}[n]=E\left\{x^{m}[n+k]\right\}\right.$

\subsubsection{Processo estocástico estacionário no sentido estrito}

O processo aleatório $X(t)$ é dito estacionário no sentido estrito ou estritamente estacionário se: $F X(t 1+\tau), X(t 2+\tau), \ldots, X(t k+\tau)(x 1, x 2, \ldots, x k)=F X(t 1), X(t 2), \ldots, X(t k)(x 1, x 2, \ldots, x k)$, quaisquer que sejam $\tau, k$ e os instantes de observação $t 1, \ldots, t k$.

\subsubsection{Condições de estacionalidade}

Para que haja as condições ideias de estacionalidade é preciso observar três condições:

1. A média do processo deverá ser constante: $\langle x[n]\rangle=\bar{x}$

2. A autocorreção do processo $R_{x}[k]$ só dependerá do valor da constante $\mathrm{K}$.

3. A variância do processo seja finita: $\sigma_{x}^{2}=\left\langle x^{2}[n]\right\rangle-\bar{x}^{2}<\infty$

Sendo a seguinte fórmula usada para o sinal contínuo $-\mathrm{x}(\mathrm{t})$

$R_{x}(\tau) \stackrel{T F}{\leftrightarrow} P_{x}(j w)=\int_{-\infty}^{+\infty} E\left[x\left[(t) x^{*}(t-\tau)\right] e^{-j w \tau} d \tau\right.$

E a seguinte fórmula usada para o sinal discreto $-x[t]$

$R_{x}(k) \stackrel{T F}{\leftrightarrow} P_{x}\left(e^{j \Omega}\right)=\sum_{-\infty}^{+\infty} E\left[x[n] x^{*}[n-k]\right] e^{-j \Omega k}$

\subsection{Processos estocásticos ergódicos.}

Definimos um processo estocástico como ergódico se as médias conjuntas forem iguais às médias temporais (Sá, 2001).

Ou seja, se:

$\left\langle x^{m}[n]\right\rangle=E\left\{x^{m}[n]\right\}$ 
Para os processos ergódicos, as médias estatísticas podem ser obtidas por meio de medias temporais realizadas a partir de uma única função amostra, ou seja, "ao longo" do processo (Guimarães, 2007).

Um processo será classificado como ergódico se apresentar as seguintes propriedades:

- A média $\bar{x}=\langle x[n]\rangle=E\{x[n]\}$ é a componente contínua (DC) do sinal $x[n]$.

- O quadrado da média, $\bar{x}^{2}$, é a potência DC.

- A média quadrada, $\overline{x^{2}}=\left\langle x^{2}[n]\right\rangle=E\left\{x^{2}[n]\right\}$, é a potência média do sinal.

- A variância $\sigma_{x}^{2}=\overline{x^{2}}-\overline{x^{2}}$ é a potência relativa à parte do sinal que varia no tempo, ou seja, sem componente DC.

- O desvio padrão $\sigma_{x}$ é o valor eficaz do sinal

\subsection{FUNÇÕES DE CORRELAÇÃO}

\subsubsection{Função de correlação cruzada.}

Esta função é par e mede a correlação entre a variável aleatória gerada por amostragem do processo $\mathrm{X}(\mathrm{t})$ em um instante $\mathrm{t}$ qualquer e a variável aleatória gerada por amostragem do processo $\mathrm{Y}(\mathrm{t})$ em um instante $\mathrm{t}+\tau$. Sua principal aplicação reside na verificação do grau de ortogonalidade entre processos aleatórios: dois processos estocásticos são ditos ortogonais se a função de correlação cruzada é nula.

\subsubsection{Função de auto correlação.}

A função de auto correlação de um processo aleatório $X(t)$ é a relação das variáveis aleatórias $X(\mathrm{t} 1)$ e $X(\mathrm{t} 2)$, obtidas pela observação do processo aleatório nos instantes $\mathrm{t} 1$ e $\mathrm{t} 2$, respectivamente.

Com isso constatamos que a função auto correlação de segunda ordem de um processo estacionário no sentido estrito depende somente da diferença de tempo.

Se a função de auto correlação de um processo aleatório é nula, significa que as variáveis obtidas por amostragem deste processo nos instantes t1 e t2 são descorrelacionadas. Neste caso a correlação entre estas variáveis passará a ser determinada pelo produto das médias do processo em questão, avaliadas nos instantes t1 e t2 (Guimarães, 2007).

Tabela 1: Relação da correlação cruzada e a auto correlação

\begin{tabular}{|l|l|l|}
\hline \multirow{2}{*}{ Correlação cruzada } & Sinais Discretos & $R_{x y}[k]=E\left\{x[n] y^{*}[n-k]\right\}$ \\
\cline { 2 - 3 } & Sinais Contínuos & $R_{x y}(\tau)=E\left\{x(t) y^{*}(t-\tau)\right\}$ \\
\hline \multirow{2}{*}{ Auto correlação } & Sinais Discretos & $R_{x}[k]=E\left\{x[n] x^{*}[n-k]\right\}$ \\
\cline { 2 - 3 } & Sinais Contínuos & $R_{x}(\tau)=E\left\{x(t) x^{*}(t-\tau)\right\}$ \\
\hline
\end{tabular}




\subsubsection{Propriedades de auto correlação.}

A função de auto correlação deve apresentar as seguintes propriedades:

- $R_{x}[-k]=R_{x}[k]-F u n c ̧ a ̃ o P a r$

- $R_{x}[0]=\overline{x^{2}}=\sigma_{x}^{2}+\bar{x}^{2} \geq R_{x}[k]$

- De 1 e 2 pode-se concluir que $R_{x}[k]$ é uma função par com um máximo em $k=0$

- Em processos não periódicos $\lim _{k \rightarrow+\infty} R_{x}[|k|]=\bar{x}^{2}$

- No caso de processos periódicos, a auto-correlação é também periódica, com o mesmo período que o processo.

\subsection{FILTROS FIR DE WIENER}

O filtro FIR de Wiener produz uma estimativa do erro quadrático médio mínimo do processo $d[n]$ filtrando o processo estatisticamente relacionado a $x[n]$.

Assume-se que os processos $x[n]$ e $d[n]$ são estacionários em sentido restrito com:

- $\quad$ Auto-correlação de $x[n]-R_{x}[k]$

- $\quad$ Auto-correlação de $d[n]-R_{d}[k]$

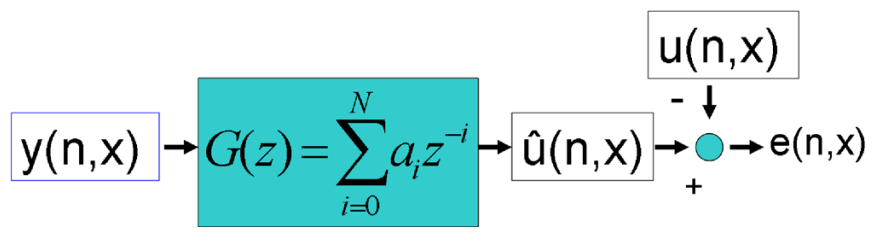

- $\quad$ Correlação cruzada: $\mathrm{R}_{\mathrm{dx}}[\mathrm{k}]$

Figura 2: Diagrama de Blocos do Filtro de Wiener (Muñoz, Antonio, Mosquera \& Alberto, 2010):

\subsubsection{Propriedades do filtro FIR de Wiener.}

O filtro FIR de Wiener assume as seguintes propriedades:

1. $\quad N$-Comprimento de filtro FIR de Wiener $W(z)=\sum_{k=0}^{N-1} w[k] z^{-k}$

2. A estimativa do filtro à saída é dada pela convolução

$$
d[n]=\sum_{t=0}^{N-1} w[l] x[n \sim l]
$$

3. O filtro de FIR de Wiener leva a coeficientes $w[n]$ que minimiza o erro quadrático médio, ou seja:

$$
\xi=E\left\{|e[n]|^{2}\right\}=E\left\{|d[n]=\hat{d}[n]|^{2}\right\} \Rightarrow \frac{\partial \xi}{\partial \omega[m]}=0
$$

\subsubsection{Princípio da ortogonalidade aplicado ao filtro FIR de Wiener.}

Manipulando matematicamente a função, obtemos: 
$\frac{\partial \xi}{\partial \omega[m]}=0 \Rightarrow E\left\{e[n] x^{*}[n-m]\right\}=0, m=0,1, \ldots, N-1$

Considerando:

$e[n]=d[n]-\hat{d}[n]=d[n]-\sum_{l=0}^{N-1} \omega[l] x[n-l]$

Resulta em:

$E\left\{d[n] x^{*}[n-m]\right\}-\sum_{l=0}^{N-1} \omega[l] E\left\{x[n-l] x^{*}[n-m]\right\}=0$

Ou seja:

$\sum_{l=0}^{N-1} \omega[l] R_{x}[m-l]=R_{d x}[m], m=0,1, \ldots, N-1$

Considerando que $R_{x}[k]=R_{x}^{*}[-k]$ resulta um sistema de equação na forma matricial (Equação de Winer-Hopf): $R_{x}=R_{d x} \Leftrightarrow w=R_{x}^{-1} R_{d x}$ Chegamos a seguinte matriz de correção:

$R_{x}=\left[\begin{array}{ccc}R_{x}[0] & R_{x}^{*}[1] & R_{x}^{*}[N-1] \\ R_{x}[1] & R_{x}[0] & R_{x}^{*}[N-2] \\ R_{x}[2] & R_{x}[1] & R_{x}^{*}[N-3] \\ \vdots & \vdots & \vdots \\ R_{x}[N-1] & R_{x}[N-2] & R_{x}[0]\end{array}\right] w=\left[\begin{array}{c}w[0] \\ w[1] \\ w[2] \\ \vdots \\ w[N-1]\end{array}\right] R_{d x}=\left[\begin{array}{c}R_{d x}[0] \\ R_{d x}[1] \\ R_{d x}[2] \\ \vdots \\ R_{d x}[N-1]\end{array}\right]$

Onde:

- $\boldsymbol{R}_{\boldsymbol{x}}$ - Matriz de auto-correlação, hermitiana e de "Toeplitz"

- $\boldsymbol{W}$-Vetor dos coeficientes do filtro

- $\boldsymbol{R} \boldsymbol{d x}$ - Vetor de correção cruzada entre o sinal desejado d[n] e o sinal observado x[n].

Gerando as seguintes equações para o filtro FIR de Wiener:

1 - Equações de Wiener-Hopf

$\sum_{l=0}^{N-1} w[l] R_{x}[m-l]=R_{d x}[m], m=0,1, \ldots, N-1$

2 - Equações de Correlações

$R_{x}=E\left\{x[n] x^{*}[n-m]\right\}$

$R_{d x}=E\left\{d[n] x^{*}[n-m]\right\}$

3 - Equações de Mínimo Erro

$\xi_{\text {min }}=R_{d}(0)-\sum_{l=0}^{N-1} w[l] R_{d x}^{*}[l]$

$w=R_{x}^{-1} R_{d x} \Rightarrow \xi_{\text {min }}=R_{d}(0)-R_{d x}^{H} R_{x}^{-1} R_{d x}$ 


\section{MODELO COMPUTACIONAL}

Para a observar os conceitos de Ergodicidade e Autocorreção aplicados ao filtro de Wiener foi desenvolvido um modelo computacional onde foi gerado um sinal desejado (sinal de referência) com uma frequência sinusoidal de 0,45 e 200 amostras. E um sinal desejado para tratar um sinal de ruído branco com distribuição normal e uma variância igual a 1. A ideia é recuperar um sinal defeituoso, ou seja, com ruído. Para isso foi calculada a matriz " $R$ " e o vetor " $p$ ", para obter " $w$ ". Onde aplicou-se os conceitos de Wiener para obtenção dos termos que estarão diretamente ligados aos parâmetros de finalização de um filtro de recuperação mais ótimo possível.

Logo em seguida observou-se o sinal após a filtragem através do filtro recém calculado, e o comparou com o sinal desejado.

Finalmente para compreender o tipo de filtragem realizado pelo filtro de Wiener, observou-se a resposta do filtro contra o espectro do sinal recebido, sendo constatado que o espectro do sinal após a filtragem é bom.

Enfim, verificou-se que o modelo atenda aos quesitos de recuperação de um sinal ruidoso.

$\mathrm{W}=\mathrm{inv}\left(\mathrm{R}^{\prime *} \mathrm{R}\right) * \mathrm{R}^{\prime *} \mathrm{p}$

$\mathrm{Na}$ tabela abaixo estão expostos os valores de " $w$ " para serem utilizados no processo de tratamento do sinal aqui atribuído. Onde o vetor " $\mathrm{p}$ " utilizado computacionalmente é referente ao vetor " $\mathrm{R}$ " do desenvolvimento matemático.

\begin{tabular}{|l|l|l|l|}
\hline \multicolumn{4}{|c|}{ Tabela 2: Valores de "w" do modelo trabalhado } \\
\hline 0.1160 & 0.0918 & 0.0451 & 0.0093 \\
\hline-0.0383 & -0.0789 & -0.1253 & -0.1083 \\
\hline-0.1142 & -0.0576 & -0.0094 & 0.0411 \\
\hline 0.0729 & 0.1107 & 0.1214 & \\
\hline
\end{tabular}

\subsection{GRÁFICOS DE ANÁLISE DE DESEMPENHO}

O gráfico abaixo mostra o comportamento do sinal corrompido com o ruído $x[n]$.

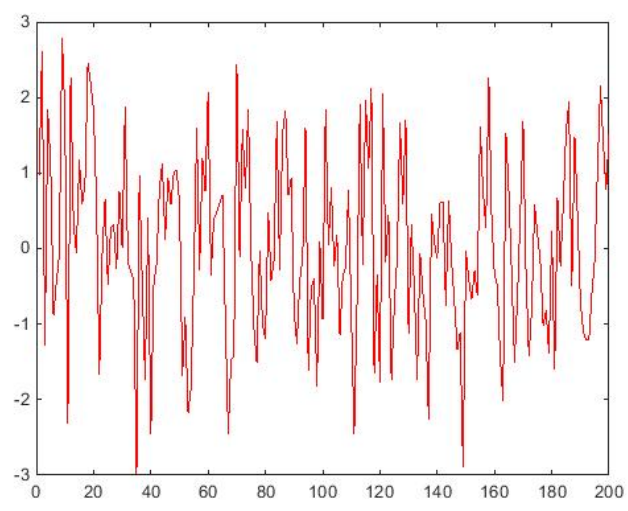




\section{Figura 3: Sinal corrompido $x[n]$}

O próximo gráfico mostra o comportamento do sinal original $\mathrm{d}[\mathrm{n}]$ e o sinal corrompido com o ruído $x[n]$, os quais são sobrepostos para que possamos ter uma ideia de como ambos estão relacionados, e como deveremos tratar este sinal.

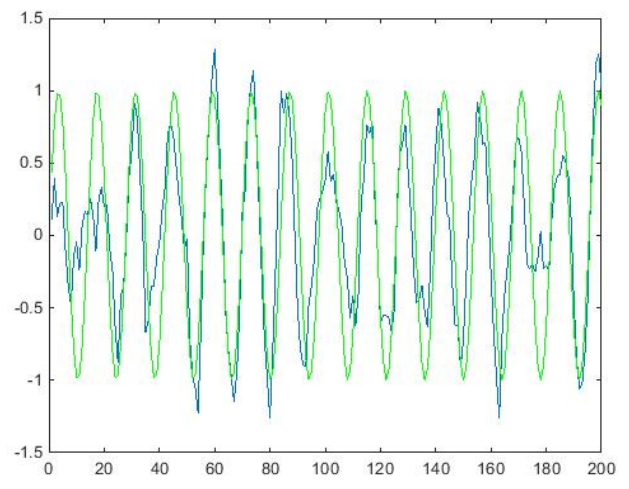

Figura 4: Sinal corrompido $x[n]$ e sinal original $d[n]$

Em sequência, o sinal corrompido mostrado na figura 3 e 4 foi exposto a o filtro de rejeição de faixa de Wiener.

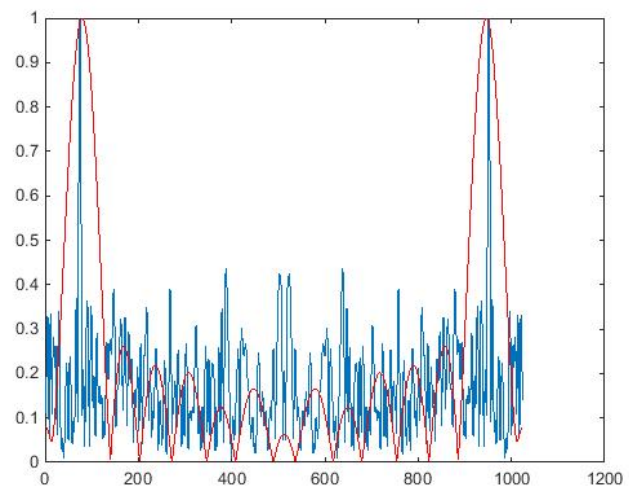

Figura 5: Sinal após a filtragem e o filtro recém-calculado

Em seguida obteve-se o seguinte resultado após a filtragem, onde foi comparado o sinal de origem, o qual desejamos aperfeiçoar, com o sinal desejado, tornando livre de grande parte do ruído adquirido no processo simulado. 


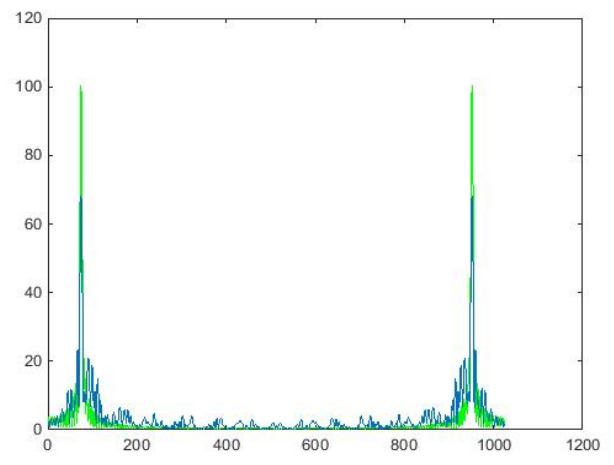

Figura 6: Espectro do sinal de origem após a filtragem e comparado com o sinal desejado

\section{RESULTADOS}

Observou-se através do modelo computacional aplicado ao sinal ruidoso mostrado na figura 3, que o comportamento obtido na simulação torna a aplicação aceitável, pois o espectro do sinal após a aplicação do filtro apresenta um bom comportamento ao ser comparado com o sinal desejado.

Finalmente, podemos concluir que a aplicação dos conceitos de Ergodicidade a Autocorreção empregados ao filtro de Wiener traz efeitos satisfatórios principalmente se forem empregados na recuperação de sinais adquiridos em ambientes ruidosos, no enriquecimento da qualidade de imagem ou ainda, para restauração de gravações antigas.

\section{CONCLUSÕES}

No ponto de vista teórico notamos a importância dos conceitos Ergódicos de um modo global para as aplicações atribuídas com esta ferramenta matemática, de certa forma as melhoras obtidas foram significativas e demonstraram a vantagem na utilização do processamento de restauração do sinal com ruído, através da aplicação do filtro de Wiener.

Com esses procedimentos aplicados notaram-se situações em que os conceitos teóricos aqui fundamentados, são de extrema importância para o desenvolvimento do método aqui desenvolvido.

Conforme a proposta deste trabalho, o filtro de Wiener, melhora o sinal gerado com ruído e fornece um desempenho melhor na restauração do sinal, onde verificamos o comportamento nos gráficos aqui expostos, auxiliando na determinação da existência de soluções ótimas para recuperação de sinais.

\section{REFERÊNCIAS}

Davila, V. H. L. (2009) Introdução a séries temporais. Notas de Aula. Disponível em <http://www.ime.unicamp.br/ hlachos/MaterialSeries.pdf > Acesso em 13 nov. 2015.

Filho, S. N. (2009) Fundamentos sobre ruídos. Disponível em: <http://www.etelj.com.br/etelj/artigos/ 151280402b0bc1accfaea913d6301caf.pdf> Acesso em 
13 nov. 2015.

Guimarães, D. A. (2007) TP501 - Processos Aleatórios. Notas de Aula. Disponível em <http://www.inatel.br/docentes/dayan/easyfolder/TP501/TP501_Notas_Processos_ Aleatorios.pdf> Acesso em 13 nov. 2015.

Lueg, P. (1932); “Process of silencing sound oscillations”. US Patent No. 2043416.

Muñoz, V.; Antonio, J.; Mosquera, S.; Alberto, J. (2010) Estimativa da atenuação ultrassônica em estruturas biológicas mediante a aplicação do filtro de wiener Anais da 9th Brazilian Conference on Dynamics Control and their Applications - ISSN 2178-3667, Serra Negra-SP. Disponível em <http://www.sbmac.org.br/ dincon/trabalhos/PDF/biological/68026.pdf> Acesso em 13 nov. 2015.

Oliveira, M. A. (2007) Sinais e ruídos. Disponível em: http://marcioandreyoliveira.blogspot.com.br/2007/08/sinais-e-rudos-conceitos-bsicos.html Acesso em 13 nov 2015.

Portal Action. (2015) Processos Estacionários. Disponível em: <http://www.portalaction.com.br/series-temporais/13-processos-estacionario>. Acesso em 13 nov. 2015.

Sá, J. P. M. (2001) Processamento de Sinal, Notas de Aula. Disponível em <https://web.fe.up.pt/ $\sim$ jmsa/apsi/APSI-3.PDF> Acesso em 13 nov. 2015.

Sant'anna, M. M. (2008) Sources of noise.

Sarmento, C. F.; Regis, D.; Araujo, W. (2012) Remoção Adaptativa de Ruídos em Sinais de Áudio, Anais do VII CONEPI, Palmas-TO Disponível em: <http://propi.ifto.edu.br/ocs/index.php/connepi/vii/paper/viewFile/2986/2540> Acesso em 13 nov. 2015.

Silva, M. A. G. (2009) Filtros digitais aplicados em sinais de Áudio. Dissertação de mestrado, Universidade Federal de Juiz de Fora. 